\title{
Colorimetric Reverse Transcription-Loop-Mediated Isothermal Amplification Assay for Rapid Detection of SARS-CoV-2
}

\author{
Meng Yee Lai, ${ }^{1}$ Soo Nee Tang, ${ }^{2}$ and Yee Ling Lau ${ }^{1 *}$ \\ ${ }^{1}$ Department of Parasitology, Faculty of Medicine, University of Malaya, Kuala Lumpur, Malaysia; ${ }^{2}$ Department of Medical Microbiology, \\ Faculty of Medicine, University of Malaya, Kuala Lumpur, Malaysia
}

\begin{abstract}
Coronavirus disease 2019 (COVID-19), which is caused by severe acute respiratory syndrome coronavirus 2 (SARS-CoV-2), has been spreading rapidly all over the world. In the absence of effective treatments or a vaccine, there is an urgent need to develop a more rapid and simple detection technology of COVID-19. We describe a WarmStart colorimetric reverse transcription-loop-mediated isothermal amplification (RT-LAMP) assay for the detection of SARSCoV-2. The detection limit for this assay was $1 \mathrm{copy} / \mu \mathrm{L}$ SARS-CoV-2. To test the clinical sensitivity and specificity of the assay, 37 positive and 20 negative samples were used. The WarmStart colorimetric RT-LAMP had $100 \%$ sensitivity and specificity. End products were detected by direct observation, thereby eliminating the need for post-amplification processing steps. WarmStart colorimetric RT-LAMP provides an opportunity to facilitate virus detection in resourcelimited settings without a sophisticated diagnostic infrastructure.
\end{abstract}

The outbreak of a cluster of respiratory illness caused by severe acute respiratory syndrome coronavirus 2 (SARSCoV-2) has infected millions of people globally. The disease was named coronavirus disease 2019 (COVID-19) and it was declared by the $\mathrm{WHO}^{1}$ as a pandemic as the virus spread rapidly throughout the world. The virus has strong personto-person transmissibility and is spread through droplets produced from coughing, saliva, and sneezing. ${ }^{2}$

Early and rapid diagnostic assays for SARS-CoV-2 is important to reduce the spread of this virus because there is currently a lack of effective antiviral drugs. To date, TaqMan probe based-real-time qualitative polymerase chain reaction (PCR) is the most popular molecular diagnostic method used for the detection of SARS-CoV-2. However, real-time qualitative $\mathrm{PCR}$ requires expensive equipment and has a long turnover time. ${ }^{3}$

In an attempt to mitigate this weakness, we developed a faster and visible loop-mediated isothermal amplification (LAMP) assay for the diagnosis of SARS-CoV-2 infection. The LAMP is a simple and rapid detection method that synthesizes a large amount of nucleic acid within 30 minutes without the need for complicated and expensive equipment.

For rapid and direct visualization of the end products in our study, we used a commercially available colorimetric LAMP reagent for the detection of COVID-19 samples. This is a two-color reverse transcription (RT)-LAMP assay protocol for detecting SARS-CoV-2 viral RNA. The inclusion of $\mathrm{pH}$-indicator dye phenol red in the master mix enables a change in color from pink to yellow if a positive amplification occurs. A no-template control was always included.

A total of 57 nasopharyngeal swabs samples from a recent COVID-19 outbreak in Malaysia (March 2020) were obtained from the University of Malaya Medical Center and were examined using the WarmStart colorimetric RT-LAMP assay. The samples were confirmed previously as COVID-19 by real-time RT-PCR prior to using the WarmStart colorimetric RT-LAMP assay. Of 57 samples, 37 tested positive by realtime RT-PCR and 20 tested negative. Ethics approval was

*Address Correspondence to Yee Ling Lau, Department of Parasitology, Faculty of Medicine, University of Malaya, 50603 Kuala Lumpur, Malaysia. E-mail: lauyeeling@um.edu.my obtained from the University of Malaya Medical Research Ethics Committee (202041-8418).

The WarmStart colorimetric RT-LAMP assay was conducted using primers targeted on the RNA-dependent RNA polymerase $(R d R p)$ gene. Primers were designed using Primer-Explorer V4 software (Eiken Chemical Co., Ltd., Tokyo, Japan) based on the SARS-CoV2 RdRp marker (GenBank accession nos. LR757995.1, LR757998.1, and MT192773.1). The LAMP assay was performed in a $25-\mu \mathrm{L}$ reaction mixture that consisted of $12.5 \mu \mathrm{L}$ WarmStart colorimetric LAMP $2 \times$ master mix (New England Biolabs, Ipswich, MA) at $65^{\circ} \mathrm{C}$ in a Loopamp Real-Time Turbidimeter LA 500 (Eiken Chemical Co.). Positive and negative reactions were indicated by yellow and pink colors, respectively (Figure 1).

A recombinant plasmid carrying the $R d R p$ gene was constructed to test the analytical sensitivity of the WarmStart colorimetric RT-LAMP assay. RdRp F3 and B3 primers were used to amplify the RdRp gene from a synthetic fragment (Sangon Biotec Co., Ltd., Shanghai, China). PCR conditions were as follows: denaturation at $94^{\circ} \mathrm{C}$ for 3 minutes, 30 cycles at $94^{\circ} \mathrm{C}$ for 30 seconds, at $55^{\circ} \mathrm{C}$ for 30 seconds, and at $72^{\circ} \mathrm{C}$ for 30 minutes, and a final extension step at $72^{\circ} \mathrm{C}$ for 10 minutes. The PCR product was then subjected to $2 \%$ agarose gel electrophoresis. The amplified gene fragment was purified prior to cloning into the pGEM-T vector (Promega Corporation, Madison, WI) and transformed into TOP10F' Escherichia coli-competent cells. Recombinant plasmids were extracted using the Qiagen Spin Miniprep kit (Qiagen, Hilden, Germany) and were sent to MyTACG Biosciences Enterprise (Kuala Lumpur, Malaysia) for sequencing to confirm its identity. The pGEM-T vector containing the $R d R p$ insert was linearized by $B a m H I$ and transcribed to RNA using RiboMAX ${ }^{\mathrm{TM}}$ Large Scale RNA Production Systems (Promega Corporation, Madison, WI) according to the manufacturer's instructions. The RNA copy number was calculated based on the following formula: copies $/ \mu \mathrm{L}=6.02 \times$ $10^{23} \times 10^{-9} \times$ concentration $(\mathrm{ng} / \mu \mathrm{L}) /($ fragment length $\times$ 340). ${ }^{4}$ Then, 10 -fold serial dilutions of the transcribed RNA ranging from $1 \times 10^{6}$ copies $/ \mu \mathrm{L}$ to $1 \mathrm{copy} / \mu \mathrm{L}$ were prepared. Results show that the limit of detection of the transcribed RNA was 1 copy/ $\mu \mathrm{L}$.

The specificity of the WarmStart colorimetric RT-LAMP assay was tested using genomic RNA of coronaviruses 


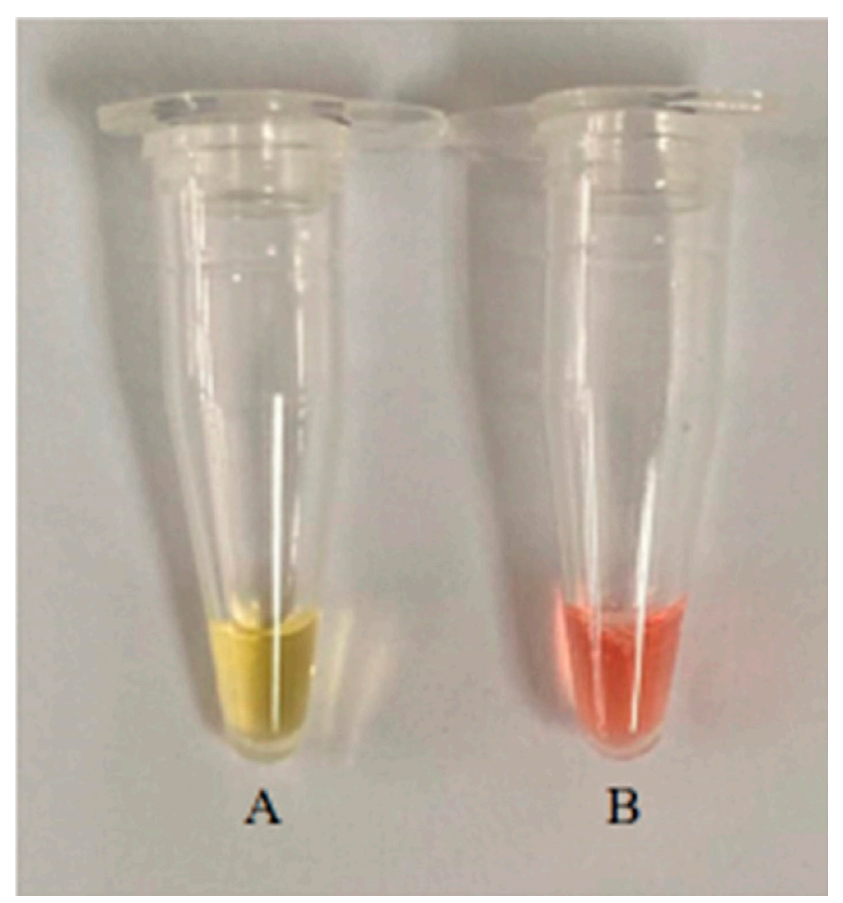

FIGURE 1. Detection of loop-mediated isothermal amplification (LAMP) end product by color changes. (A) Positive amplified LAMP product changed to yellow color. (B) Negative amplified LAMP product remained red. This figure appears in color at www.ajtmh.org.

(HCoV-OC43 and SARS-CoV), adenovirus, human metapneumovirus, influenza $A(A / H 1 p d m 2009$ and $A / H 3)$ and influenza $B$ viruses, parainfluenza virus 3 , rhinovirus $A$, respiratory syncytial virus $B$, and enterovirus D68. The results show the specificity was $100 \%$.

The clinical sensitivity and specificity of the WarmStart colorimetric RT-LAMP were evaluated using 57 samples collected from the University of Malaya Medical Center. Sensitivity was calculated as (number of true positives)/(number of true positives + number of false negatives); specificity was calculated as (number of true negatives)/(number of true negatives + number of false positives). The WarmStart colorimetric RT-LAMP detected all the real-time RT-PCR positive samples as positive and did not detect any of the real-time RT-PCR negative samples. It had $100 \%$ sensitivity and specificity.

Compared with other studies, our study shows that the lowest detection limit of the RT-LAMP assay was 1 copy/ $\mu \mathrm{L}$ within 30 minutes by using the $R d R p$ gene as a target. Huang et al., ${ }^{5}$ on the other hand, showed that the lowest detection limit of a colorimetric assay was 2 copies/ $\mu \mathrm{L}$ within 30 minutes by using the $S$ gene. Their findings also showed that the lowest detection limit for the Orf1ab gene was 20 copies/ $\mu \mathrm{L}$. Baek et al. $^{6}$ managed to detect the $N 1$ gene down to 100 copies/ $\mu \mathrm{L}$ within 30 minutes by using the WarmStart colorimetric RT-LAMP assay.

We evaluated the use, suitability, and advantages of the WarmStart colorimetric RT-LAMP assay for the detection of SARS-CoV-2. Cross-contamination can be reduced because monitoring using the WarmStart colorimetric RT-LAMP assay relies on color changes of the end products. Thus, no post-amplification work such as gel electrophoresis and ethidium bromide gel staining are required. The $\mathrm{pH}$ indicator dye was incorporated in the reaction buffer during manufacture, thereby avoiding the need for additional dye for viewing end products after amplification. The risk of carryover contamination was also reduced because the cap of the tube was not opened, which minimizes the opportunity of airborne aerosol being released into surrounding areas.

Some measures of precaution need to be considered when handling RNA samples because they degrade rapidly and need to be "reverse transcripted" to complementary DNA prior to proceeding with any downstream work. The WarmStart colorimetric RT-LAMP assay is a one-step protocol for detecting RNA. Additional reverse transcriptase enzyme did not need to be added to the reaction mixture because it was included in the reaction buffer during manufacture. This is another advantage of this assay. Coupled with these advantages, this reagent has been used by other investigators as well. Ahn et al. ${ }^{7}$ used this reagent successfully to detect influenza viruses $(\mathrm{H} 1 \mathrm{~N} 1, \mathrm{H} 3 \mathrm{~N} 2$, and Type $\mathrm{B})$ and avian influenza viruses ( $\mathrm{H} 5 \mathrm{~N} 1, \mathrm{H} 5 \mathrm{~N} 6, \mathrm{H} 5 \mathrm{~N} 8$, and $\mathrm{H} 7 \mathrm{~N} 9)$, with a $98.9 \%$ positive rate compared with $92.3 \%$ when RT-PCR was used. With this similar reagent, Thi et al. ${ }^{8}$ achieved $99.5 \%$ specificity and $86 \%$ sensitivity during their development of a swab-to-RT-LAMP assay for detecting SARS-CoV-2 RNA in clinical samples.

\section{CONCLUSION}

The results of our study suggest that the WarmStart colorimetric RT-LAMP assay may provide a rapid, sensitive, and reliable diagnostic method for detecting SARS-CoV-2 without the need for sophisticated equipment. The assay may be used as an initial screening tool, followed by confirmatory laboratory testing such as the conventional real-time RT-PCR method. The WarmStart colorimetric RT-LAMP assay may potentially increase the speed of diagnosis and overcome the rapid spread of SARS-CoV-2.

Received February 7, 2021. Accepted for publication May 10, 2021.

Published online June 15, 2021.

Acknowledgments: The following reagent was obtained through the National Institutes of Health Biodefense and Emerging Infections Research Resources Repository, National Institute of Allergy and Infectious Diseases: gamma-irradiated SARS-coronavirus, NR-9547. The American Society of Tropical Medicine and Hygiene has waived the Open Access fee for this article due to the ongoing COVID-19 pandemic.

Financial support: This study was supported by the Prototype Research Grant Scheme (PR001-2020B) from the Ministry of Education, Malaysia.

Authors' addresses: Meng Yee Lai and Yee Lin Lau, Department of Parasitology, Faculty of Medicine, University of Malaya, Kuala Lumpur, Malaysia, E-mails: mengylai11@yahoo.com and lauyeeling@ um.edu.my. Soo Nee Tang, Department of Medical Microbiology, Faculty of Medicine, University of Malaya, Kuala Lumpur, Malaysia, E-mail: tang.soonee@ummc.edu.my.

This is an open-access article distributed under the terms of the Creative Commons Attribution (CC-BY) License, which permits unrestricted use, distribution, and reproduction in any medium, provided the original author and source are credited.

\section{REFERENCES}

1. World Health Organization, 2020. Novel Coronavirus (2019-nCoV) Situation Report, 10. Geneva, Switzerland: WHO. Available at: 
https://apps.who.int/iris/handle/10665/330775. Accessed May 24, 2021.

2. Shereen MA, Khan S, Kazmi A, Bashir N, Siddique R, 2020. COVID-19 infection: origin, transmission, and characteristics of human coronaviruses. J Adv Res 24: 91-98.

3. Lu R, Wu X, Wan Z, Li Y, Zuo L, Qin J, Jin X, Zhang C, 2020. Development of a novel reverse transcription loop-mediated isothermal amplification method for rapid detection of SARSCoV-2. Virol Sin 35: 344-347.

4. Yan C et al., 2020. Rapid and visual detection of 2019 novel coronavirus (SARS-CoV-2) by a reverse transcription loopmediated isothermal amplification assay. Clin Microbiol Infect 26: 773-779.
5. Huang WE et al., 2020. RT-LAMP for rapid diagnosis of coronavirus SARS-CoV-2. Microb Biotechnol 13: 950-961.

6. Baek YH et al., 2020. Development of a reverse transcriptionloop mediated isothermal amplification as a rapid earlydetection method for novel SARS-CoV-2. Emerg Microbes Infect 9: 998-1007.

7. Ahn SJ et al., 2020. Rapid and simple colorimetric detection of multiple influenza viruses infecting humans using a reverse transcriptional loop mediated isothermal amplification (RT-LAMP) diagnostic platform. BMC Infect Dis 19: 676.

8. Thi DVL et al., 2020. A colorimetric RT-LAMP assay and LAMPsequencing for detecting SARS-CoV-2 RNA in clinical samples. Sci Transl Med 12: eabc7075. 